\title{
R-Process Nucleosynthesis in MHD Jet Explosions of Core-Collapse Supernovae
}

\author{
Motoaki Saruwatari, ${ }^{1}$ Masa-aki Hashimoto, ${ }^{1}$ Ryohei Fukuda, ${ }^{1}$ and Shin-ichiro Fujimoto ${ }^{2}$ \\ ${ }^{1}$ Department of Physics, Kyushu University, Hakozaki, Fukuoka 812-8581, Japan \\ ${ }^{2}$ Department of Electronic Control, Kumamoto National College of Technology, Kumamoto 861-1102, Japan \\ Correspondence should be addressed to Masa-aki Hashimoto; hashimoto@phys.kyushu-u.ac.jp
}

Received 30 March 2013; Accepted 26 August 2013

Academic Editors: W. Cui, Y.-Z. Fan, and J. F. Valdés-Galicia

Copyright (c) 2013 Motoaki Saruwatari et al. This is an open access article distributed under the Creative Commons Attribution License, which permits unrestricted use, distribution, and reproduction in any medium, provided the original work is properly cited.

We investigate the $r$-process nucleosynthesis during the magnetohydrodynamical (MHD) explosion of a supernova in a helium star of 3.3 $M_{\odot}$, where effects of neutrinos are taken into account using the leakage scheme in the two-dimensional (2D) hydrodynamic code. Jet-like explosion due to the combined effects of differential rotation and magnetic field is able to erode the lower electron fraction matter from the inner layers. We find that the ejected material of low electron fraction responsible for the $r$-process comes out from just outside the neutrino sphere deep inside the Fe-core. It is found that heavy element nucleosynthesis depends on the initial conditions of rotational and magnetic fields. In particular, the third peak of the distribution is significantly overproduced relative to the solar system abundances, which would indicate a possible $r$-process site owing to MHD jets in supernovae.

\section{Introduction}

Study of the $r$-process has been developed considerably keeping pace with the terrestrial experiments of nuclear physics far from the stability line of nuclides [1]. In particular, among the three peaks, which correspond to the elements of ${ }^{80} \mathrm{Se},{ }^{130} \mathrm{Te}$, and ${ }^{195} \mathrm{Pt}$, in the abundance pattern for the solar system $r$-elements, the transition from the second to third peak elements has been stressed by nuclear physicists [2]. Although supernovae could be one of the astrophysical sites of the $r$-process $[2,3]$, explosion mechanism is not still completely resolved, where supernova explosions are originated from the gravitational collapse of massive stars of $M \geq 8 M_{\odot}[4,5]$. However it is unclear whether neutronrich elements could be ejected or not during the shock wave propagation. As far as the one-dimensional calculations, almost all realistic numerical simulations concerning the collapse-driven supernovae of $M \geq 13 M_{\odot}$ have failed to explode the outer layer above the Fe-core due to drooping of the energetic shock wave propagation [6,7]. Although there exist calculations for 8 and $11 M_{\odot}$ stars to explode, the explosion energies are very weak [8-15]. Therefore, a plausible site/mechanism of the $r$-process has not yet been clarified.
On the other hand, models of magnetorotational explosion (MRE) for core-collapse supernovae have been presented as a supernova mechanism [16-19] since both rapid rotations and/or strong magnetic fields could be resulted for neutron stars after the explosions. Furthermore, MRE with a realistic magnetic field configuration has been investigated [20-22]. In their series of papers, it has been shown that magnetorotational instability plays a critical role concerning the explosion energies which would explain the explosion energies of Type II and Ib supernovae. However, changes in the electron fractions and/or the heavy element nucleosynthesis have not been discussed well. Therefore, it should be studied whether MHD explosions affect the $r$-process even within a qualitative method.

Two-dimensional (2D) magnetohydrodynamical (MHD) calculations have been performed with the various initial parameters concerning rotation and magnetic field [13, 2327]. The ZEUS-2D code [28] has been modified to include an equation of state [29], electron captures with a simple scheme of neutrino ( $\nu$-) transport [23]. Adopting these achievements, Nishimura et al. [30] have performed 2D/MHD calculations to study possibilities of the $r$-process during the supernova explosion of massive stars under the assumption of adiabatic 
explosion. They have shown the pattern of distributions of the $r$-elements of the solar system abundances. However, they have also found that the electron fractions $\left(Y_{e}\right)$ increase significantly enough to destroy the $r$-process elemental distributions if the neutrino capture processes are included, where the processes were obtained from the results of spherical explosion calculations with the realistic neutrino transport included [31]. The problem is remained whether the adopted method for neutrino captures can be legitimate or not; effects of neutrino transport have not been included at all, and instead Nishimura et al. [30] have used the profiles of densities and temperatures obtained from the adiabatic calculations. It should be done to check their results by including the neutrino transport and to study the explosion energies even under extreme parameters of rotation/magnetic fields. In the meanwhile, Winteler et al. [32] have shown the $r$-process nucleosynthesis with the use of results of a magnetorotationally driven supernova simulation, where they have performed $3 \mathrm{D}$ calculations with a $3 \mathrm{D}$ spectral scheme for neutrinos. Although they have obtained enough $r$-elements, their MHD simulation has finished at around $31 \mathrm{~ms}$ after the bounce. It would be useful to show results of two-dimensional MHD calculations with longer simulation time and/or higher resolution for calculations of the $r$-process. It is noted that Winteler et al. [32] have emphasized the possible importance of the early appearance of $r$-process matter in low metallicities which could be originated by MHD explosion of supernovae.

In the present paper, we give the calculational results of the MHD explosion for the He-star of $3.3 M_{\odot}$ untill the final simulation time $t_{f} \simeq 600 \mathrm{~ms}$. Although these results have already been briefly reported [33], we show the details of simulation procedure and their results to compensate the contents. For the MHD calculations, five models are adopted for the initial configuration of rotation and magnetic fields. In the initial magnetic field, we assume very strong magnetic fields which validity is not known. In the observations of magnetar with the magnetic field $1.6 \times 10^{14} \mathrm{G}$ [34], they have suggested a rather massive progenitor mass from the age of all the early type stars. In the complete online magnetar catalog cited by Mori et al. [34], magnetic field of 26 magnetars ranges $10^{14}-10^{16} \mathrm{G}$ which have been obtained from the analysis of the decrease in the rotational period under the assumption of magnetic dipole braking in a vacuum. These observations encourage us to set the initial condition of a strong magnetic field. Contrary to the previous investigation of the $r$-process under adiabatic MHD explosion [30], we include the effects of neutrinos using a leakage scheme [35-40] with some modifications explained in Section 2. Finally, we investigate the possibility of the $r$-process in the MHD jets with use of our large nuclear reaction network. We find the region that produces the $r$-process elements having the particular distribution of low $Y_{e}$.

In Section 2, our supernova models that include the neutrino effects are given, and we also explain the initial models and $r$-process networks. The results of the $r$-process nucleosynthesis calculations are presented in Section 3. We summarize our results in Section 4, discuss remained problems, and propose future works in Section 5.

\section{Supernova Models}

2.1. MHD Equations. Ideal MHD equations are enumerated as follows $[13,39]$ :

$$
\begin{gathered}
\frac{d \rho}{d t}+\rho \nabla \cdot \mathbf{v}=0 \\
\rho \frac{d \mathbf{v}}{d t}=-\nabla P-\rho \nabla \Phi+\frac{1}{4 \pi}(\nabla \times \mathbf{B}) \times \mathbf{B}, \\
\frac{\partial \mathbf{B}}{\partial t}=\nabla \times(\mathbf{v} \times \mathbf{B}), \\
\nabla^{2} \Phi=4 \pi G \rho \\
\rho \frac{d(e / \rho)}{d t}=-P \nabla \cdot \mathbf{v}+Q^{+}-Q^{-}
\end{gathered}
$$

where $\rho$ is the density, $\mathbf{v}$ is the velocity, $P$ is the pressure, $\mathbf{B}$ is the magnetic field, $e$ is the internal energy density, and $Q^{+}$and $\mathrm{Q}^{-}$are the neutrino $(\nu)$ heating and cooling rates, respectively. The gravitational potential $\Phi$ is solved from the Poisson solver [28].

2.2. Neutrino Leakage Scheme. Neutrino luminosity $\left(L_{\nu}\right)$ at a neutrino sphere can be estimated from the average $\nu$-energy $\bar{\epsilon}_{\nu, \text { esc }}$ (see (15) later) that escapes freely:

$$
L_{v}=\int_{V} \bar{\epsilon}_{\nu, \mathrm{esc}} \frac{n_{\nu}}{\tau_{\mathrm{esc}}} d V,
$$

where $n_{v}$ is the $\nu$-number density and $\tau_{\text {esc }}$ is the escape time for a neutrino to reach the $v$-sphere $R_{v}$, that is, obtained from the leakage scheme $[38,41]$ in terms of the $\nu$-mean free path $\left(\lambda_{\text {tot }}\right)$ defined by

$$
\int_{R_{v}}^{\infty} \frac{1}{\lambda_{\text {tot }}} d r=\frac{2}{3} .
$$

The mean free path of neutrinos is given as

$$
\lambda_{\text {tot }}^{-1}=\frac{\rho Y_{N}}{m_{u}} \sigma_{\mathrm{coh}}+\frac{\rho Y_{p}}{m_{u}} \sigma_{\mathrm{sc}, p}+\frac{\rho Y_{n}}{m_{u}} \sigma_{\mathrm{sc}, n}+\frac{\rho Y_{n}}{m_{u}} \sigma_{a b},
$$

where $m_{u}$ is the atomic mass unit and $Y_{n}, Y_{p}$, and $Y_{N}$ are the number fractions relative to baryons for neutrons, protons, and nuclei, respectively. The values of $\sigma_{\mathrm{sc}, p}, \sigma_{\mathrm{sc}, n}$, and $\sigma_{\mathrm{coh}}$ are the cross sections for scattering on protons, neutrons, and nuclei, respectively.

Contrary to the original leakage scheme, we do not adopt free stream approximation for neutrinos outside $R_{v}$. Since the minimum size of the mesh interval in our hydrodynamic calculations is $10^{5} \mathrm{~cm}$, we prescribe the time step so that for each time step of $\Delta t$, neutrinos run by $c \Delta t$, which is typically $10^{4} \mathrm{~cm}$ in our simulations.

The terms of $Q^{+}$and $Q^{-}$are calculated in the following $[42,43]$. Outside the neutrino sphere, the two terms are, respectively,

$$
\begin{gathered}
Q^{+}=\sigma_{a b} n_{n} F_{v}, \\
Q^{-}=\Gamma_{p} n_{p}+\Gamma_{n} n_{n}+R_{e e}+R_{\gamma},
\end{gathered}
$$


where $n_{n}\left(n_{p}\right), F_{v}$, and $\sigma_{a b}$ are the number density of free neutrons (protons), energy flux of neutrinos at each point calculated by the equation of continuity, and the $v$-absorption cross section by free neutrons $\left(n+v_{e} \rightarrow p+e^{-}\right)$, that is, the most important heating source and a function of average energy and density, respectively [44]. Neutrino production (emission) rates, $\Gamma_{p}, \Gamma_{n}$, and $R_{e e}, R_{\gamma}$ are explained below (see (8) and the equations below (18)). Inside the neutrino sphere, we calculate only the term $Q^{-}$as follows:

$$
Q^{-}=\bar{\epsilon}_{v, \mathrm{esc}} \frac{n_{v}}{\tau_{\mathrm{esc}}} .
$$

For the $\bar{\nu}$-absorption by free protons, we can get both $Q^{+}$ and $Q^{-}$by replacing physical quantities between $v$ and $\bar{\nu}$.

2.3. Neutrino Processes and Physical Inputs. The change in electron fraction $Y_{e}$ is given by (e.g., Kotake et al. [39])

$$
\frac{d Y_{e}}{d t}=-\Gamma_{p} Y_{p}-\Gamma_{N} Y_{N}-\frac{c}{\lambda_{\nu}} Y_{n}+\frac{c}{\lambda_{\bar{v}}} Y_{p}
$$

where $\lambda_{\nu}$ is the mean free path of electron neutrino and $\lambda_{\bar{\nu}}$ is that of antineutrino [44]. The last two terms in the right hand side, which manifest the effects of $v$-radiation, play an essential role to change $Y_{e}$ after around $200 \mathrm{~ms}$ measured from the bounce.

The electron capture rate by a proton $\left(p+e^{-} \rightarrow n+v_{e}\right)$ with $Q_{p}=1.3 \mathrm{MeV}$ is obtained from Epstein and Pethick [45]:

$$
\begin{aligned}
& \Gamma_{p}=\frac{1}{2 \pi^{3} \hbar} \frac{G_{F}^{2} C_{V}^{2}\left(1+3 a^{2}\right)}{\left(h^{3} c^{3}\right)^{2}} I_{p}, \\
& I_{p}=\int_{Q}^{\mu_{e}} d E_{e} E_{e}^{2} E_{\nu}^{m} f_{e}\left(1-f_{\nu}\right) .
\end{aligned}
$$

Here $m=2, Q=Q_{p}+\mu_{\nu}$, and $f_{\alpha}$ is the Fermi-Dirac distribution of particles $\alpha \quad(=e, v)$ :

$$
f_{\alpha}=\frac{1}{1+e^{\left(\epsilon_{\alpha}-\mu_{\alpha}\right) / T_{\alpha}}},
$$

where $\epsilon_{\alpha}$ and $\mu_{\alpha}$ in units of the Boltzmann constant are the single particle energy and the chemical potential, respectively. We note that outside the equilibrium region between neutrinos and baryons; significant thermal deviation comes out between temperatures of neutrinos and baryons, that is, $T_{v} \neq T_{m}$. The fundamental constant of $G_{F}$ is the Fermi coupling, $C_{V}=0.97$ the pseudovector coupling, and $C_{A}$ the axial vector coupling ones. We set the ratio $\left|C_{A} / C_{V}\right|=1.27$. The electron capture rate by a nucleus of the atomic number $Z$ with the $Q$-value $Q_{N}$ is

$$
\Gamma_{N}=\frac{12}{7} \frac{1}{2 \pi^{3} h} \frac{G_{F}^{2} C_{A}^{2}(Z-20)}{\left(h^{3} c^{3}\right)^{2}} I_{N},
$$

where $I_{N}$ is obtained from (9) with the values of $m=2$ and $Q=Q_{N}+\mu_{v}$. Note that this capture process is assumed to be inhibited above the neutron number $N=40$ due to the effects of shell blocking [45-47]. We should note that the temperature effects and correlations are responsible for removing the inhibition [47].

Energies of emitted neutrinos by the individual electron captures are given by

$$
E_{\nu, p}=\frac{J_{p}}{I_{p}}, \quad E_{v, N}=\frac{J_{N}}{I_{N}},
$$

where $J_{p}$ and $J_{N}$ are obtained from $I_{p}$ and $I_{N}$ with $m=3$, respectively $[45,48]$. The average energy of neutrinos emitted by electron captures is written as follows:

$$
\bar{\epsilon}_{v}=\frac{E_{\nu, p} \dot{Y}_{p}+E_{\nu, N} \dot{Y}_{N}}{\dot{Y}_{p}+\dot{Y}_{N}} .
$$

This average energy is added to obtain the $v$-energy density at the next time step of simulations.

Inside the $\nu$-sphere, $T_{\nu}$ and $\mu_{\nu}$ are evaluated in terms of $n_{\nu}$ and the $v$-energy density $e_{v}$ :

$$
\begin{aligned}
& n_{\nu}=\frac{T_{v}^{3}}{(\hbar c)^{3}} \int 4 \pi \epsilon_{v}^{2} f_{v} d \epsilon_{v}, \\
& e_{\nu}=\frac{T_{v}^{4}}{(\hbar c)^{3}} \int 4 \pi \epsilon_{\nu}^{3} f_{v} d \epsilon_{v},
\end{aligned}
$$

where the Fermi-Dirac distribution is assumed for neutrinos. Outside the $v$-sphere, neutrino radiation can be approximated to be the black body one with $\mu_{\nu}=0$. The average $\nu$-energy is written as follows;

$$
\bar{\epsilon}_{\gamma, \mathrm{esc}}=\frac{F_{3}}{F_{2}} T_{\nu, \mathrm{sp}}
$$

where $T_{\gamma, \text { sp }}$ is the $\nu$-temperature at the $v$-sphere and the Fermi integrals, $F_{2}$ and $F_{3}$, are, respectively:

$$
F_{2}=\int_{0}^{\infty} \frac{x^{2}}{1+e^{x}} d x, \quad F_{3}=\int_{0}^{\infty} \frac{x^{3}}{1+e^{x}} d x
$$

We can approximate these terms by the analytic formula given by Epstein and Pethick [45]. Since $T_{\nu} \geq T_{m}$ inside the $\nu$-sphere, the baryon energy density $e_{m}$ increases due to the energy flow from neutrinos:

$$
\frac{d e_{m}}{d t}=\frac{c}{\lambda_{v}} e_{v}
$$

where $\lambda_{v}$ is the mean free path of relevant neutrinos [44]. In this region, we replace $T_{\gamma, \mathrm{sp}}$ by $T_{m}$ to obtain $\bar{\epsilon}_{\nu, \mathrm{esc}}$ in (15), because neutrino temperature decreases due to the diffusive effect around the $v$ sphere, which would underestimate the $v$ energy. This is our first modification of the original leakage scheme.

The similar procedure for the positron capture rate $\Gamma_{n}$ can be applied to anti-neutrinos of the reaction, $n+e^{+} \rightarrow p+\bar{v}_{e}$, with the substitutions

$$
\mu_{e} \longrightarrow-\mu_{e}, \quad \mu_{v} \longrightarrow \mu_{\bar{\nu}}, \quad T_{v} \longrightarrow T_{\bar{v}} .
$$


Other neutrino processes are as follows:

$$
\begin{gathered}
e^{+}+e^{-} \longrightarrow v_{x}+\overline{\nu_{x}} \\
\gamma \longrightarrow v_{x}+\overline{\nu_{x}} \\
x=e, \tau, \mu .
\end{gathered}
$$

Here, $x$ means electron, $\tau$, and $\mu$-neutrinos. These processes are important for a late stage of the explosion, because the most neutrino luminosity for the late stage of the explosion comes from these processes. The rates of these processes have been obtained by Ruffert et al. [36, 37]. While electron neutrinos emitted by these processes contribute to neutrino cooling and heating, $\mu, \tau$-neutrinos do only neutrino cooling. The emission rate of $v_{e}$ or $\overline{\nu_{e}}$ by electron-positron pair annihilation is given by

$$
\begin{aligned}
R_{e e}\left(\nu_{e}, \overline{\nu_{e}}\right)= & \frac{\left(C_{1}+C_{2}\right)_{\nu_{e}, \overline{\nu_{e}}}}{36} \frac{\sigma_{0} c}{\left(m_{e} c^{2}\right)^{2}} \epsilon_{e^{-}} \cdot \epsilon_{e^{+}} \\
& \times\left(1-f_{v_{e}}(\epsilon)\right)_{e e}\left(1-f_{\overline{\nu_{e}}}(\epsilon)\right)_{e e},
\end{aligned}
$$

where $\sigma_{0}=1.76 \times 10^{-44} \mathrm{~cm}^{2}$, and $\epsilon_{e^{-}}$or $\epsilon_{e^{+}}$indicates electron/positron energy density. The weak interaction constants are $\left(C_{1}+C_{2}\right)=\left(C_{V}-C_{A}\right)^{2}+\left(C_{V}+C_{A}\right)^{2}$, and $(1-$ $\left.f_{v_{e}}(\epsilon)\right)_{e e}$ is the blocking factor in the neutrino phase space and approximately expressed by

$$
\begin{aligned}
(1 & \left.-f_{v_{e}}(\epsilon)\right)_{e e} \\
& =\left(1+\exp \left(-\left(\frac{1}{2} \frac{F_{4}\left(\eta_{e}\right)}{F_{3}\left(\eta_{e}\right)}+\frac{1}{2} \frac{F_{4}\left(-\eta_{e}\right)}{F_{3}\left(-\eta_{e}\right)}-\eta_{v_{e}}\right)\right)\right)^{-1},
\end{aligned}
$$

where $F_{n}(\eta)$ means fermi integral:

$$
F_{n}(\eta)=\frac{T^{n+1}}{(\hbar c)^{n+1}} \int_{\eta}^{\infty} \frac{x^{n}}{1-e^{x-\eta}} d x
$$

with $\eta=\mu_{e} / T$.

For the production of $\nu_{\mu}, \overline{\nu_{\mu}}$ and $\nu_{\tau}, \overline{\nu_{\tau}}$, the corresponding rate is

$$
\begin{aligned}
R_{e e}\left(v_{x}\right)= & \frac{\left(C_{1}+C_{2}\right)_{v_{x}, \overline{\nu_{x}}}}{9} \frac{\sigma_{0} c}{\left(m_{e} c^{2}\right)^{2}} \\
& \times \epsilon_{e^{-}} \cdot \epsilon_{e^{+}}\left(1-f_{v_{x}}(\epsilon)\right)_{e e}^{2},
\end{aligned}
$$

where $\left(C_{1}+C_{2}\right)_{v_{x}, \bar{v}_{x}}=\left(C_{V}-C_{A}\right)^{2}+\left(C_{V}+C_{A}-2\right)^{2}$.

The rate of creation of $\nu_{e}$ or $\bar{\nu}_{e}$ by the decay of transversal plasmons can be written with sufficient accuracy as

$$
\begin{aligned}
R_{\gamma}\left(\nu_{e}, \overline{\nu_{e}}\right)= & \frac{\pi^{3}}{3 \alpha^{*}} C_{V}^{2} \frac{\sigma_{0} c^{2}}{\left(m_{e} c^{2}\right)^{2}} \frac{T^{8}}{(h c)^{6}} \gamma^{6} \exp (-\gamma) \\
& \times(1+\gamma)\left(1-f_{\nu_{e}}(\epsilon)\right)_{\gamma}\left(1-f_{\overline{\nu_{e}}}(\epsilon)\right)_{\gamma}
\end{aligned}
$$

and the corresponding rate for producing $v_{x}$ becomes

$$
\begin{aligned}
R_{\gamma}\left(\nu_{x}\right)= & \frac{4 \pi^{3}}{3 \alpha^{*}}\left(C_{V}-1\right)^{2} \frac{\sigma_{0} c^{2}}{\left(m_{e} c^{2}\right)^{2}} \frac{T^{8}}{(h c)^{6}} \gamma^{6} \\
& \times \exp (-\gamma)(1+\gamma)\left(1-f_{v_{x}}\right)_{\gamma} .
\end{aligned}
$$

The fine structure constant, $\alpha^{*}=1 / 137.036$, and $\gamma=$ $5.5565 \times 10^{-2} \sqrt{(1 / 3)\left(\pi^{2}+3 \eta_{e}^{2}\right)}$, and $\left(1-f_{\nu_{x}}\right)_{\gamma}$ is the blocking factor,

$$
\left(1-f_{v_{x}}\right)_{\gamma}=\left[1+\exp \left(-\left(1+\frac{1}{2} \frac{\gamma^{2}}{1+\gamma}-\eta_{v_{x}}\right)\right)\right]^{-1} .
$$

2.4. Neutrinos outside the Neutrino Sphere. Neutrino number density $n_{v}$ on each mesh can be calculated as follows:

$$
\frac{d n_{v}}{d t}=\Gamma_{p} n_{p}+\Gamma_{n} n_{n}+R_{e e}+R_{\gamma}-n_{\gamma, \mathrm{esc}}
$$

where $n_{\nu \text {,esc }}$ indicates number density of protons, neutrons, and escaping neutrino density estimated by escaping time scale (see (28)), respectively. For the second modification of the leakage scheme, the last term in the right hand side in (27) is calculated in the neutrino sphere as follows:

$$
n_{v, \mathrm{esc}}=\frac{n_{v}}{\beta \tau_{\mathrm{esc}}},
$$

where $\beta \tau_{\text {esc }}$ is the neutrino escape time scale and it is estimated as follows,

$$
\tau_{\mathrm{esc}}=\max \left(\frac{\Delta R}{c}, \frac{3 \Delta R^{2}}{\pi^{2} c \lambda_{\mathrm{tot}}}\right),
$$

where $\Delta R$ is the distance from each point to the neutrino sphere $R_{v}$. The factor $\beta$ is introduced and it could take the value in the range $1-5[49,50]$. In our case, we set the value to be $\beta=2 \sqrt{3}$, considering the isotropic diffusion of neutrinos from around the neutrino sphere. The escaping neutrino density is added to the neutrino density at the neutrino sphere. Outside the neutrino sphere, we consider streaming neutrinos (see the below equation of continuity) except for slightly absorbed neutrinos. Therefore, we calculate equation of continuity:

$$
\frac{\partial n_{v}}{\partial t}+\frac{1}{r} \frac{\partial\left(n_{v} r c\right)}{\partial r}=\Gamma_{p} n_{p}+\Gamma_{n} n_{n}+R_{e e}+R_{\gamma}-n_{a b},
$$

where $n_{a b}$ is the absorbed neutrino density calculated from the heating rate $\left(Q^{+} n_{v} / e_{\nu}\right)$. We should note that Kotake et al. $[23,39]$ set the neutrino fraction to be zero outside the $\nu$-sphere. We solved the continuity equation outside the $\nu$ sphere, which affects the location of the sphere. Furthermore, they utilized a postprocessing approach for the heating term. The heating term may change the dynamics. Due to the heating, jet formation may become preferable.

In Figure 1, we show the neutrino luminosities and electron fractions calculated by the method explained in 


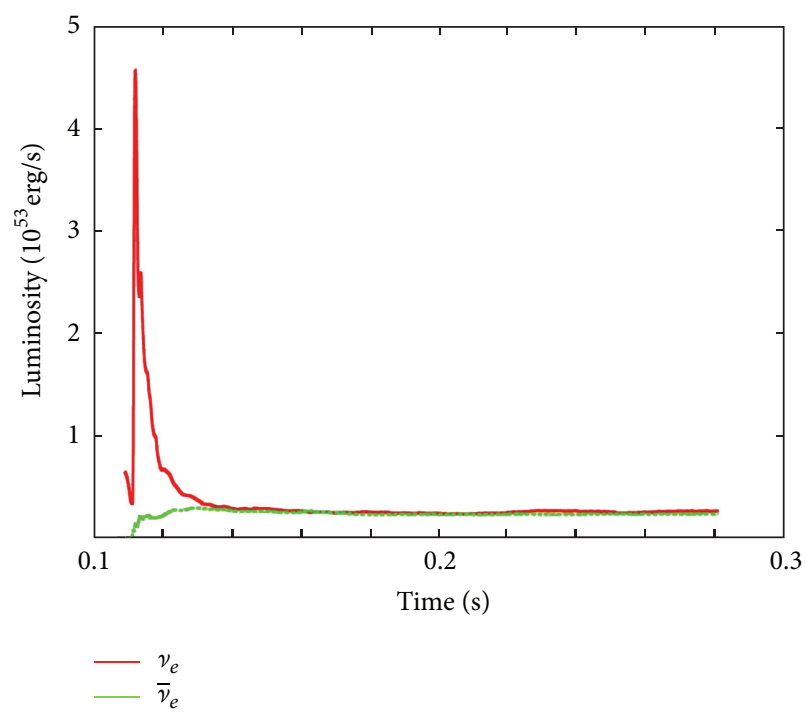

(a)

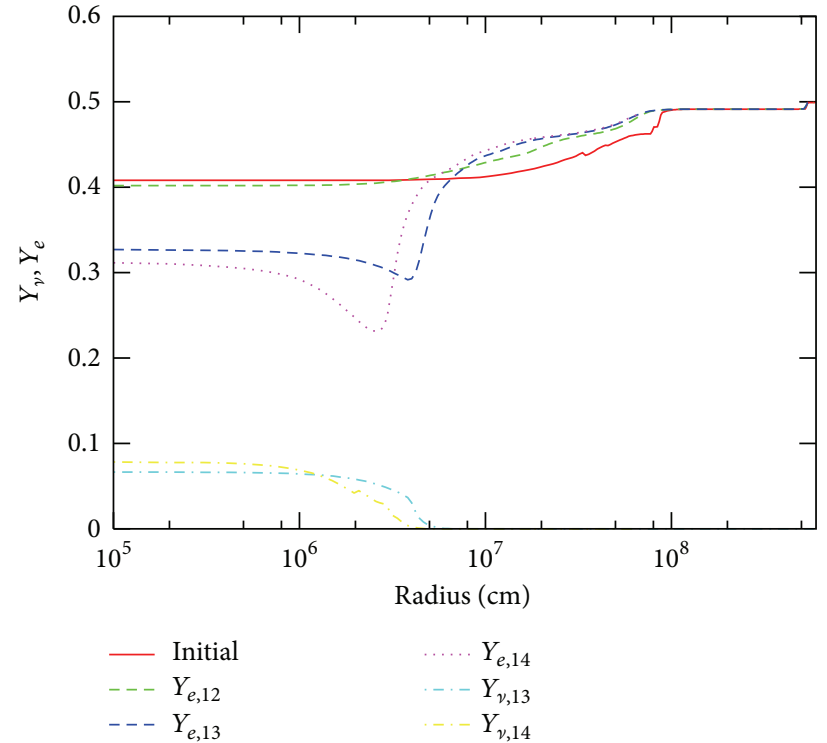

(b)

Figure 1: (a) It shows the evolution of the neutrino luminosity after the collapse for a model 1. (b) It shows changes in electron fraction $\left(Y_{e}\right)$ when the central density becomes $10^{12}, 10^{13}$, and $10^{14} \mathrm{~g} \mathrm{~cm}^{-3}$, respectively, and neutrino fraction $\left(Y_{\nu}\right)$ when the central density becomes $10^{13}$ and $10^{14} \mathrm{~g} \mathrm{~cm}^{-3}$, respectively. Our results could approximate those of Liebendörfer et al. [31].

this section which corresponds to a model of spherically symmetry (model 1 in Table 1). They are compared with the figures (Figures 8 and 1(d)) in Liebendörfer et al. [31]. Considering the simple scheme of the neutrino transport, our results could approximate more accurate ones which adopt the detailed neutrino transport scheme.

2.5. Initial Models and r-Process Networks. In all computations, spherical coordinates $(r, \theta)$ are adopted. The computational region is set to be $0 \leq r \leq 4000 \mathrm{~km}$ and $0 \leq \theta \leq \pi / 2$, where the included mass in the precollapse models amounts to $1.42 M_{\odot}$. The first quadrant of the meridian section is covered with $300(r) \times 30(\theta)$ mesh points (Fe-core plus some amounts of Silicon-rich layer).

To acquire information of mass elements, nine thousand tracer particles are distributed on each mesh point within the region of $0.449 \leq Y_{e} \leq 0.49$ from the center to the place of $1.3 M_{\odot}(r=2200 \mathrm{~km})$ at the beginning of the collapse. Five initial models are prepared as shown in Table 1 . We adopt cylindrical properties of the angular velocity $\Omega$ and the toroidal component of the magnetic field $B_{\phi}$ as follows:

$$
\begin{gathered}
\Omega(X, Z)=\Omega_{0} \frac{X_{0}^{2}}{X^{2}+X_{0}^{2}} \frac{Z_{0}^{4}}{Z^{4}+Z_{0}^{4}}, \\
B_{\phi}(X, Z)=B_{0} \frac{X_{0}^{2}}{X^{2}+X_{0}^{2}} \frac{Z_{0}^{4}}{Z^{4}+Z_{0}^{4}},
\end{gathered}
$$

where $X$ and $Z$ are the distances from the rotational axis and the equatorial plane. $X_{0}$ and $Z_{0}$ are model parameters. Both $\Omega_{0}$ and $B_{0}$ are the initial values at $X=0$ and $Z=0$. Here, we add a model 5 in addition to the initial models adopted by Nishimura et al. [30]. This model has the largest value of $\Omega_{0}$ among five models. As shown in the next section, among the five models, model 5 can eject material with very low electron fraction $Y_{e}$.

Here, we note that Winteler et al. [32] have used a shellular rotation law as their initial model of $15 M_{\odot}$ [51]. The value of $T /|W|=7.63 \times 10^{-3}$ may not be so different from those of our models. On the other hand, their initial distribution of the magnetic field is assumed to be purely poloidal field having $E_{m} /|W|=2.63 \times 10^{-8}$. The difference of the distribution of the magnetic field could affect the formation of jet along the polar direction.

Although significant improvements could have been performed for the nuclear data of nucleosynthesis calculations, we utilize the same nuclear reaction networks that have been constructed for the $r$-process calculations by Nishimura et al. [30]. This is because our purpose is to investigate some qualitative effects by including the neutrino transport on the $r$-process. Let us briefly explain the nuclear data included in the networks. The networks have been extended toward the neutron-rich side till the neutron-drip line. Each network consists of about 4000 nuclear species up to $Z=$ 100 . Included reactions are two-body ones, $(n, \gamma),(p, \gamma)$, $(\alpha, \gamma),(p, n),(\alpha, p),(\alpha, n)$, plus three-body one, $(3 \alpha, \gamma)$, and their inverses. Two kinds of the network, called FRDM and ETFSI, have been constructed. The mass formula of FRDM is constructed by the Nilsson-Struntinsky model considering effects of shell and microscopic part. ETFSI approach is a semiclassical approximation to the Hartree-Fock method in which the shell corrections are calculated with the integral version of the Strutinsky theorem. Reaction rates are constructed based on experimental data if available which are 
TABLE 1: Initial parameters of precollapse models.

\begin{tabular}{lcccccc}
\hline Model & $T /|W|(\%)$ & $E_{m} /|W|(\%)$ & $X_{0}^{*}$ & $Z_{0}^{*}$ & $\Omega_{0}\left(\mathrm{~s}^{-1}\right)$ & 0 \\
\hline Model 1 & 0 & 0 & 0 & 0 & $B_{0}(\mathrm{G})$ \\
Model 2 & 0.5 & 0.1 & 1 & 1 & 5.2 & $5.4 \times 10^{12}$ \\
Model 3 & 0.5 & 0.1 & 0.5 & 1 & 7.9 & $1.0 \times 10^{13}$ \\
Model 4 & 0.5 & 0.1 & 0.1 & 1 & 42.9 & $5.2 \times 10^{13}$ \\
Model 5 & 1.5 & 0.1 & 0.1 & 1 & 72.9 \\
\hline
\end{tabular}

Note: $X_{0}^{*}=X_{0} / 10^{8} \mathrm{~cm}$ and $Z_{0}^{*}=Z_{0} / 10^{8} \mathrm{~cm}$. Models 1 to 4 have the same initial parameters as those adopted by Nishimura et al. [30]. We add a model 5 that has the largest value of a parameter of $\Omega_{0}$ among five models, where other parameters are the same as those of the model 4.

TABLE 2: Calculated quantities that are crucial in the $r$-process.

\begin{tabular}{lccccccc}
\hline Model & $t_{b}$ & $t_{f}$ & $T /|W|_{f}$ & $E_{m} /|W|_{f}$ & $E_{\text {exp }}^{*}$ & $M_{\text {ej }} / M_{\odot}$ & $M_{r \mathrm{rj}} / M_{\odot}$ \\
\hline Model 1 & 111 & 283 & 0 & 0 & 0.023 & - & - \\
Model 2 & 125 & 311 & 6.91 & 0.053 & 0.127 & - & - \\
Model 3 & 129 & 329 & 8.74 & 0.116 & 0.164 & - & - \\
Model 4 & 133 & 433 & 8.80 & 0.142 & 1.13 & 0.111 & $5.90 \times 10^{-3}$ \\
Model 5 & 180 & 624 & 15.3 & 0.339 & 0.484 & 0.022 & - \\
\hline
\end{tabular}

Note: $t_{b}$ indicates the time (ms) at the bounce. The calculations are stopped at the time $t_{f}(\mathrm{~ms})$. The ratios $T /|W|_{f}$ and $E_{m} /|W|_{f}$ are expressed in $\% . E_{\text {exp }}^{*}=$ $E_{\text {exp }} / 10^{51}$ ergs. $M_{\mathrm{ej}}$ is the sum of the ejected tracer particles. $M_{\text {rej }}$ is the ejected mass of the $r$-element for $A \geq 63$.

supplemented by theoretical data with inverse reaction rates and partition functions with use of FRDM or ETFSI.

\section{Explosion Models, Distribution of Electron Fraction, and $r$-Process Calculations}

We investigate hydrodynamical stages of the collapse, bounce, and propagation of the shock wave with use of ZEUS-2D code using a simple neutrino transport scheme as shown in Section 2. Our results of MHD calculations are summarized in Table 2, where $E_{\exp }$ is the explosion energy when the shock reaches the edge of the Fe-core and $M_{\mathrm{ej}}$ is the mass summed over the ejected tracer particles. We note that the explosion does not occur for model 1 to model 3 . While the jet-like explosion occurs along the equator (up to $40^{\circ}$ from the equator) in model 4 , a collimated jet emerged from the rotational axis in model 5 (Figure 2). A protoneutron star remains after the jet-like explosion. During the explosion, temperature exceeds $10^{10} \mathrm{~K}$ around the original layers of the $\mathrm{Si}+\mathrm{Fe}$ core, where the nuclear statistical equilibrium is realized.

In model 4 , the equatorial region is ejected as shown in Figure 2 having rather high value of $Y_{e} \simeq 0.50$ (Figure 3(a)). In model 5 , materials are ejected with the jets along the polar regions, whose total angle is subtended over $20^{\circ}$ from the axis (see Figure 2). The corresponding evolutions of $Y_{e}$ relevant to the $r$-process are shown in Figure 4 . The lowest value of $Y_{e}<$ 0.20 is found around the polar region as seen in Figure 3(b).

Figure 5 shows the ejected mass against $Y_{e}$ in the range $0.05 \leq Y_{e} \leq 0.50$. In model 4 , the ejecta with $Y_{e}>0.40$ comes from the Si-rich layers along the equatorial region, which is attributed to the enhanced centrifugal force relative to the magnetic one. We recognize that as against the spherical explosion, $Y_{e}$ decreases significantly for model 5, due to the collimated jet along the rotational axis. This is because neutrino luminosity is low by a factor of ten compared to that of spherical explosion shown in Figure 1(a). This can be seen in Figure 6, where the density along polar axis is low compared to the case of model 4 . Therefore, the reaction responsible for the increase in $Y_{e}, n+v_{e} \rightarrow p+e^{-}$becomes ineffective.

We note that we distribute 9000 tracer particles on each mesh point. To check the change in distribution of $Y_{e}$ owing to that of tracer particles for model 5, (1) we scatter 15000 tracer particles inside the computational region and (2) nine thousand particles between $500 \mathrm{~km}$ and $2200 \mathrm{~km}$. As a result, we have confirmed that deficiency of $Y_{e}$ between $0.2-0.3$ is the same for all cases. For case (1), tiny amounts of $Y_{e}$ appears below $Y_{e}=0.2$. Therefore, nucleosynthesis results qualitatively do not depend on the method how to distribute tracer particles.

We calculate the $r$-process nucleosynthesis for the explosion model 5. Before the nucleosynthesis calculation, we have assumed abundances to be in nuclear statistical equilibrium state (NSE) as has been done $[44,52]$. The NSE code is used just after the temperature drops $10^{10} \mathrm{~K}$ to around $9 \times 10^{9} \mathrm{~K}$. Then, the nuclear reaction network of the $r$-process has been operated till the temperature decreases to $2-3 \times 10^{9} \mathrm{~K}(t \sim$ $600 \mathrm{~ms}$ ) using the results of the MHD calculations. After that, network calculations are performed until $T \sim 10^{7} \mathrm{~K}(t \sim$ $10 \mathrm{~s}$ ) with the method in Flower and Hoyle [53]. We include neutrino captures in our nucleosynthesis network that has been applied for tracer particles. The capture rates $\left(n+v_{e} \rightarrow\right.$ $\left.p+e^{-}, p+\bar{\nu}_{e} \rightarrow n+e^{+}\right)$that were not incorporated by Nishimura et al. [30] are obtained from neutrino luminosity, neutrino flux, and other hydrodynamical information as we have described in the previous sections. Concerning the nucleosynthesis, we can get actually this information after the end of NSE stage $\left(t \sim t_{f}\right)$ under the assumption of constant neutrino luminosity, since $L_{v}$ does not change appreciably 

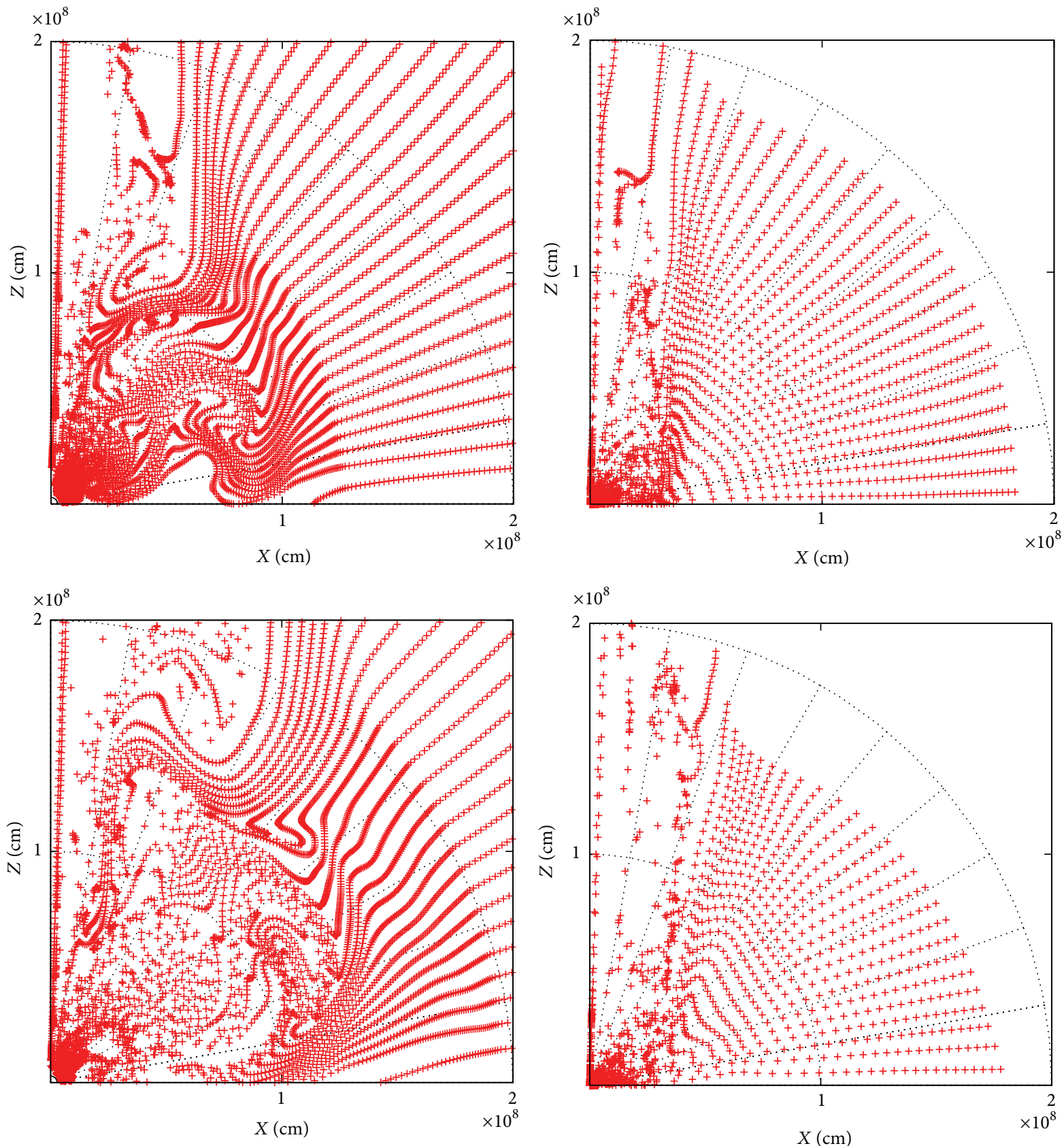

Figure 2: Snapshots of tracer particles at $t=100 \mathrm{~ms}$ (top) and $t=200 \mathrm{~ms}$ (bottom) after the bounce in models 4 (left) and 5 (right).

after $t \sim 200 \mathrm{~ms}$. We note that a difference between the neutron and proton single-particle energies in a dense medium may change neutrino capture cross sections significantly [54]. As a consequence, it is shown that the luminosities of all neutrino flavors are reduced while the spectral differences between electron neutrinos and antineutrinos are increased [55]. These changes in weak interaction processes should be examined by including them for the hydrodynamical simulations.

In case of the rapid rotation and strong magnetic field (model 4), barely jet-like explosion is obtained in the direction of the equatorial region (Figure 6(a)). It is, however, impossible to reproduce the $r$-elements even up to the second peak of the solar $r$-process abundance pattern, because $Y_{e}$ of the ejected materials distributes in the range of high values of $Y_{e} \geq 0.4$. In the model 5, where we have adopted a special initial configuration of concentrated magnetic field with strong differential rotation, jet-like explosion emerges in the direction of the rotational axis (Figure 6(b)). The difference is that model 5 has larger value of the angular velocity compared to model 4 by a factor of 1.7. We compared 


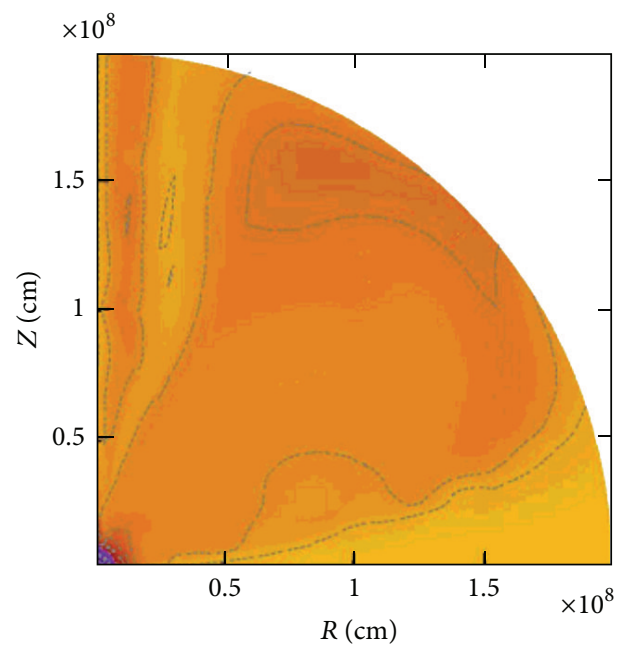

(a)

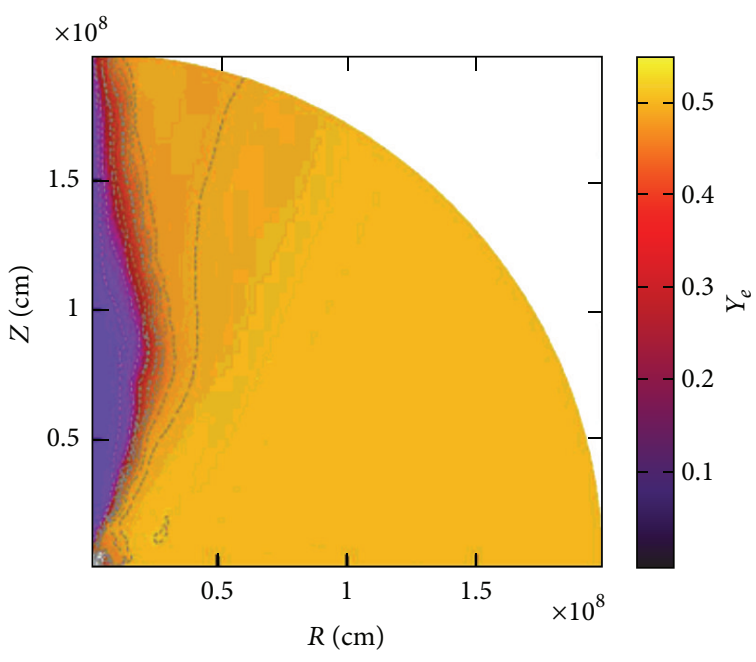

(b)

FIgURE 3: Contour of $Y_{e}$ over the range 0.1-0.5 at the final stage of calculation in model 4 (a) and model 5 (b).

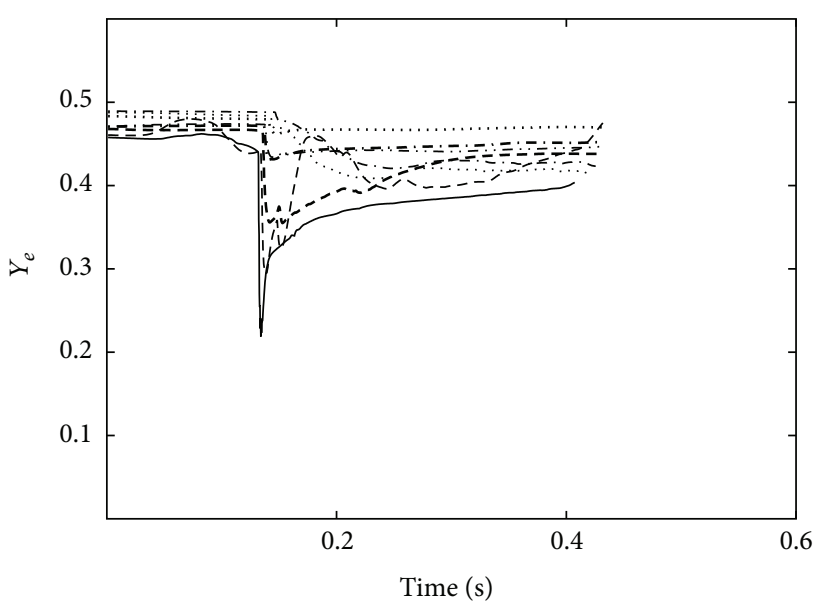

(a)

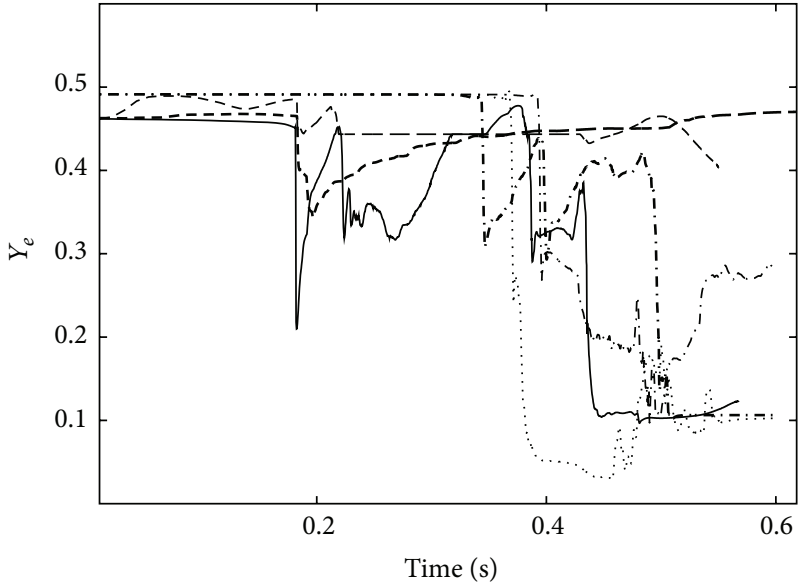

(b)

Figure 4: Time evolution of $Y_{e}$ in model 4 (a) and model 5 (b). For model 4, the explosion resembles to spherical one and all $Y_{e}$ have increased above 0.4 due to the neutrino capture process.

the produced heavy elements with the solar $r$-elements in Figure 7, where results for two different mass formulas are shown. Model 5 may present a site for reproducing only the third peak with the first and second peaks underproduced.

\section{Summary and Discussion}

We have barely shown a qualitative possibility of the $r$-process nucleosynthesis during MHD explosion in a massive star of $13 M_{\odot}$. Initial models have been constructed changing the distributions of rotation and magnetic fields parametrically [30].

We include neutrino effects by using the leakage scheme. This scheme treats neutrino effects approximately, where we assume Fermi-Dirac distribution for neutrinos. Furthermore, we add modification about the physical process just outside the neutrino sphere (15) and timescale of neutrino drift (28) and (29) in addition to the original leakage scheme. Validity for $2 \mathrm{D}$ calculations cannot be assured for this scheme, because flow to the $\theta$ direction is not included. If jets are so strong that the corresponding density becomes low, increase in $Y_{e}$ would be suppressed compared to the 1D case. Therefore, we can say that the discussion of Nishimura et al. [30] with use of an analytical formula of $1 D$ results is inaccurate.

Other schemes have been developed for solving neutrino transport [56]. For example, IDSA (Isotropic Diffusion Source Approximation) solves Boltzmann equation approximately, and the result obtained by using this scheme is consistent with one dimensional simulations, where 


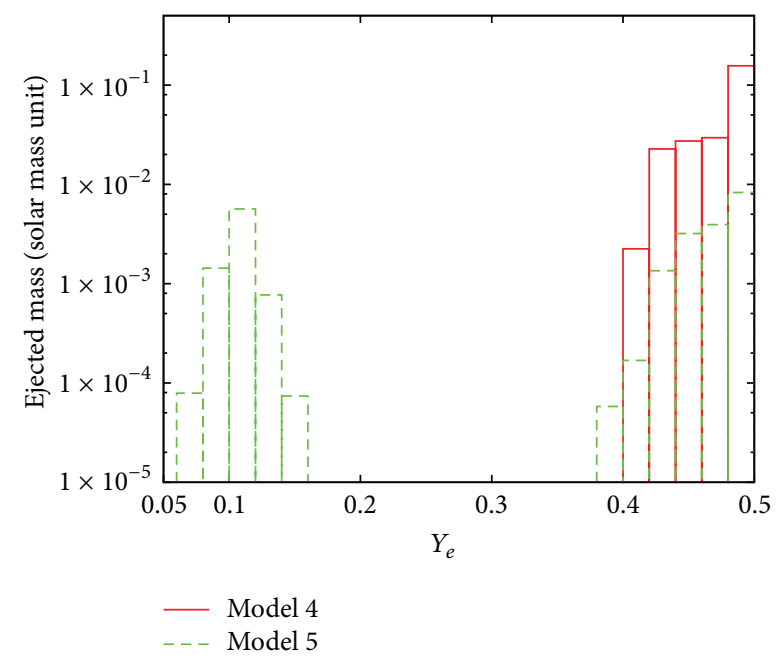

FIGURE 5: Ejected mass as a function of $Y_{e}$ at around $t_{f}=600 \mathrm{~ms}$ (model 5). The thick lines indicate the results of model $4\left(t_{f}=\right.$ $433 \mathrm{~ms}$ ). Two separate distributions in $Y_{e}$ are due to different neutrino irradiation just outside the neutrino sphere.

the Fermi-Dirac distribution has been found to be a good approximation [56].

We have calculated MHD simulations by varying two parameters in initial models (the distribution of rotation and magnetic field). The previous adiabatic simulations without neutrino effects have succeeded in explosion except for the model 3 [30]. However, in the present case, only two models with neutrino effects whose distribution of magnetic field and rotation concentrated in the central region result in explosion. Generally, for strong and concentrated rotation case with some magnetic field, a collimated jet-like explosion occurs. For relatively weak and concentrated rotation model, large region around from rotational axis to equatorial axis is blasted, which is assisted with the magnetic field effect. In the present study, ejected region is different from that of Nishimura et al. [30]. Model 5 shows that deep region is ejected in comparison with Nishimura's model 4. It means that the produced composition depends crucially on rotation parameter. Model 5 may present an appropriate site for reproducing only the third peak relative to the first and second peaks insufficiently built up. Contrary to the negative conclusion against the possible $r$-process in the previous study [30], we can show at least the elemental distributions of the $r$-elements as far as the third peak of the solar pattern is concerned. This is due to the lower neutrino luminosities from 200 to $500 \mathrm{~ms}$ after the bounce. At the same time, we have shown the possibility for the lower $Y_{e}$ materials to be ejected significantly if the neutrino transport mechanism works appropriately.

Observations of $\gamma$-ray bursts associated with supernovae are rare in the present observations [57]. Considering the relations between $\gamma$-ray bursts and the MHD explosions, the new site of the $r$-process to produce significantly only the third peak of the solar abundance pattern should be also rare. The nuclear process to produce the abundances after the third peak would have some relations to our MHD jet model. Since $\gamma$-ray bursts should have continued after the formation of the first star, a new model beyond our jet model (e.g., Winteler et al. [32]) and their motivation of the $r$ process for low metallicities would give a clue about nuclear cosmochronology represented by ${ }^{232} \mathrm{Th}$ which half life is as long as the age of the universe [52].

We could conclude that supernova explosions of massive stars associated with the $r$-process cannot be excluded under some assumed conditions; a progenitor has special distributions of rotational/magnetic fields inside the stellar core; simple neutrino transport scheme such as a leakage scheme can be applied.

\section{Future Work}

We propose that both the supernova mechanism and $r$ process nucleosynthesis still remain to be some crucial problems. This might be one of the reason why whole consistency for the origin of elements has not been understood. We should include the following effects for the $r$-process calculations.

5.1. Detailed Neutrino Transport Scheme. In the present study, we use a simple leakage scheme for description of neutrino transport. Leakage scheme can describe neutrino transport in very easy way compared to the Boltzmann equation solver. Neutrino effect, however, is very sensitive to dynamics [58], and our method may not be inadequate for the $2 \mathrm{D}$ calculations. We need to adopt a more detailed neutrino transport scheme such as that of IDSA which may be capable of applying to multi-dimensional simulations. Due to the difficulty of multi-dimensional simulations with Boltzmann equation solver, IDSA simulation will play a more important role $[13,56]$.

5.2. Neutrino Oscillation. We do not consider neutrino oscillation because transport with neutrino oscillation is difficult to handle. Since mean energies of $\tau$ and $\mu$ neutrinos are higher than those of electron neutrino, the effect may cause significant effects for neutrino heating [59]. Furthermore, it has been shown that the charged current weak interaction processes affect the luminosities of neutrino flavors which are related to neutrino oscillations [55]. These fundamental processes should be studied with hydrodynamical simulations.

5.3. SASI. Standing accretion shock instability (SASI) has been focused on hydrodynamic simulations [11-13]. It is considered to help the shock strength increase in heating regions. While SASI is in a good sense for explosion mechanism, it may play minus role for heavy element nucleosynthesis. Although a strong convection may occur behind the shock front by SASI, the convection effect tends to average the $Y_{e}$ distribution [60].

5.4. Distribution of Magnetic Field. We consider only toroidal magnetic field. If we input polar magnetic field as an initial parameter [61], even a weak rotation model may lead to 


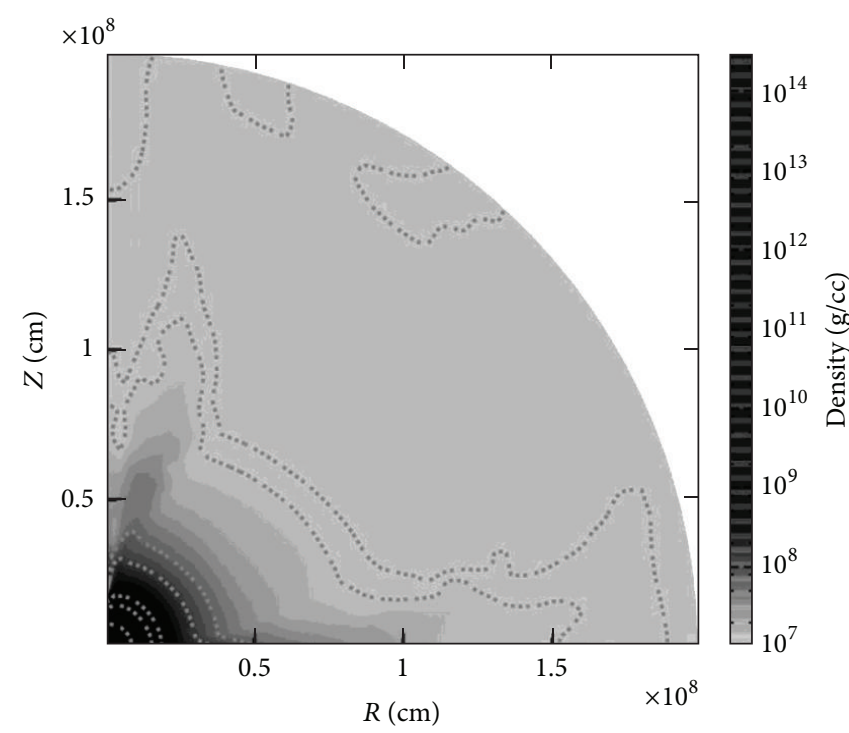

(a)

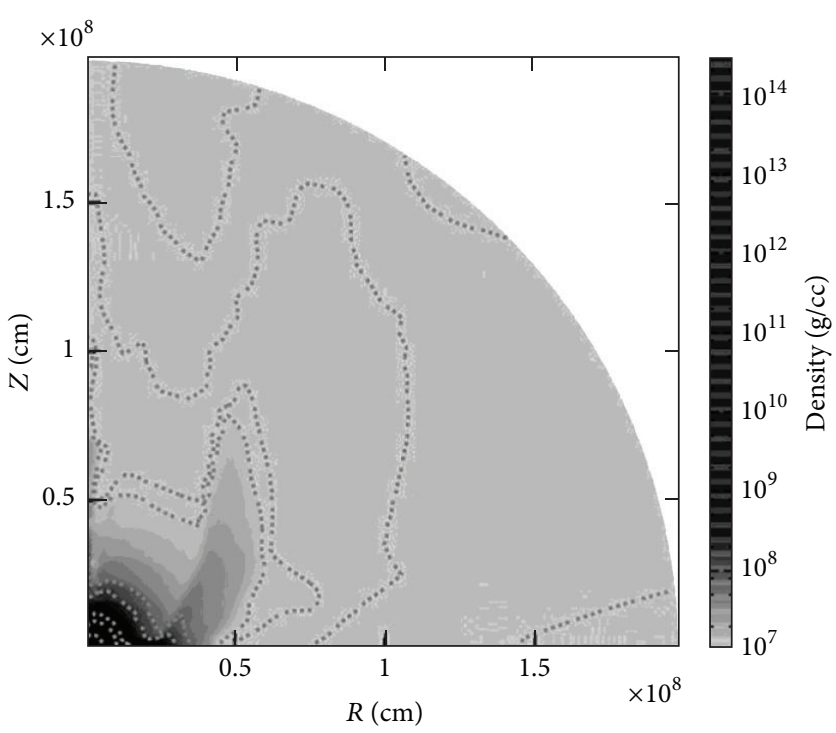

(b)

FIGURE 6: Density contour over $10^{7}-10^{14} \mathrm{~g} \mathrm{~cm}^{-3}$ at $t=200 \mathrm{~ms}$ after the bounce in model 4 (a) and model 5 (b). Model 5 indicates a jet in which density is rather low along the polar axis.

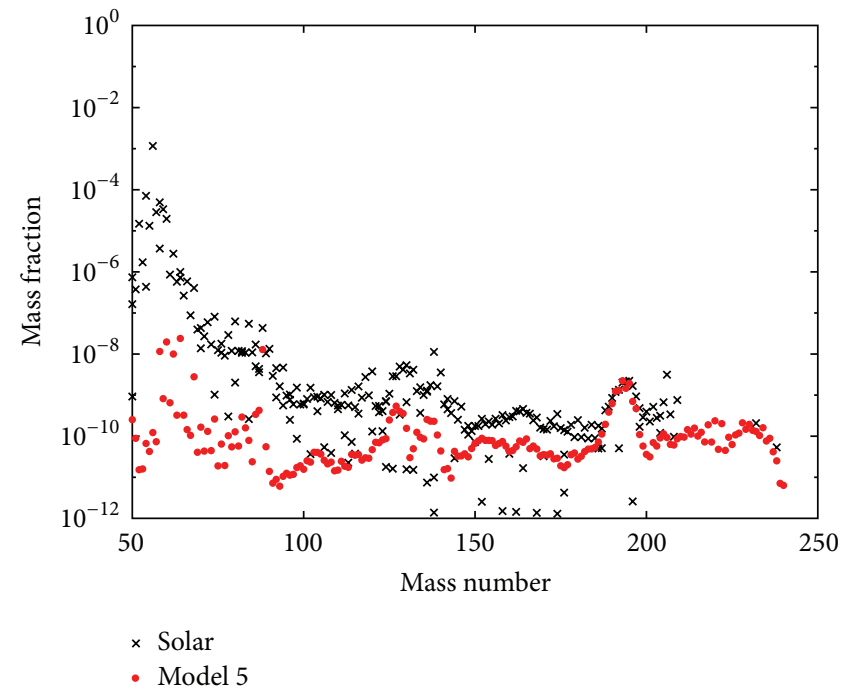

(a)

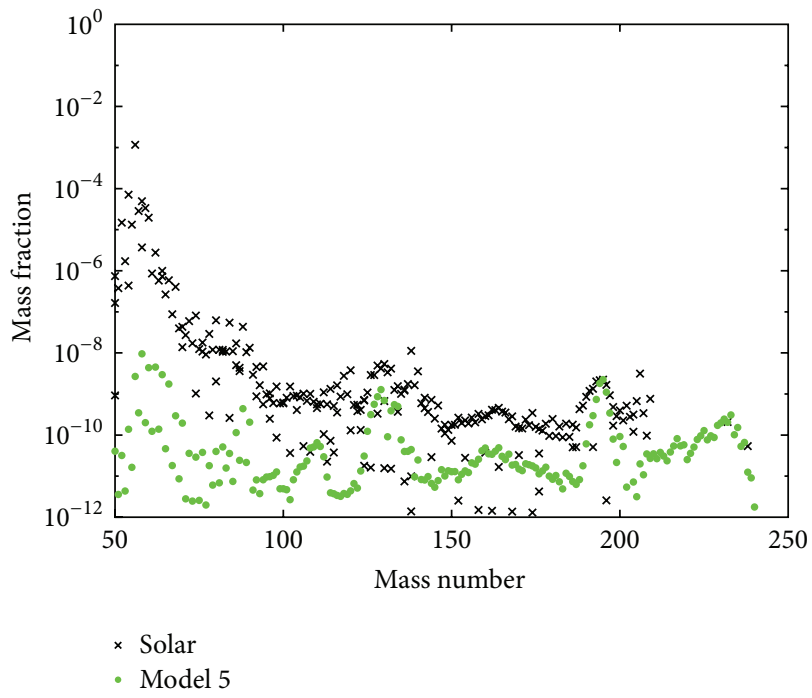

(b)

FIgURE 7: Nucleosynthesis calculation results using ETFSI (a) and FRDM (b). The third peak is reproduced for both mass formula. However, the slope of the abundance pattern of FRDM is too steep around $A \simeq 200$ to compare with the solar pattern. The last peak of $A>200$ cannot be reproduced in both cases.

succeed in explosion and draw up low $Y_{e}$ matter from deep inside the region. In this point, MRE explosion with a realistic magnetic field configuration should be investigated [20-22] to see the effects on the $r$-process.

5.5. Simulations of Other Massive Star Models. We have adopted a $3.3 M_{\odot}$ He-core model, because it has been convenient to set up an initial model. We need to simulate for higher stellar masses (e.g., Ono et al. [62]). Neutrino luminosity may rise for more massive models. Increasing neutrino luminosity could unfortunately cause neutrino capture process furthermore and heavier nuclei may not be synthesized.

5.6. Magnetar. We find that the produced protoneutron star has very strong magnetic field $\left(10^{14}-10^{16} \mathrm{G}\right)$. This might suggest the formation of magnetar [63-72]. However, in the present calculation with a numerical scheme adopted, the MRI cannot be resolved [21]; it is hopeful to study whether there exists any relation between the formation of magnetar and heavy elemental synthesis. 


\section{Acknowledgment}

This work has been supported in part by a Grant-in-Aid for Scientific Research (19104006, 21540272, 24540278, and 25400281) of the Ministry of Education, Culture, Sports, Science, and Technology of Japan. The authors would like to thank the reviewers for improving the draft.

\section{References}

[1] M. Arnould, S. Goriely, and K. Takahashi, "The r-process of stellar nucleosynthesis: astrophysics and nuclear physics achievements and mysteries," Physics Reports, vol. 450, no. 4-6, pp. 97213, 2007.

[2] F. K. Thielemann, A. Arcones, R. Käppeli et al., "What are the astrophysical sites for the $r$-process and the production of heavy elements? " Progress in Particle and Nuclear Physics, vol. 66, no. 2, pp. 346-353, 2011.

[3] Y. Z. Qian, "The r-process: current understanding and future tests," in Proceedings of the 1st Argonne/MSU/JINA/INT RIA Wokshop, 2005.

[4] M. Hashimoto, "Supernova nucleosynthesis in massive stars," Progress of Theoretical Physics, vol. 94, no. 5, pp. 663-736, 1995.

[5] A. Heger, C. L. Fryer, S. E. Woosley, N. Langer, and D. D. H. Hartmann, "How massive single stars end their life," Astrophysical Journal Letters, vol. 591, no. 1, pp. 288-300, 2003.

[6] H. T. Janka, R. Buras, K. Kifonidis, A. Marek, and M. Rampp, "Core-collapse supernovae at the threshold," Springer Proceeding in Physics, vol. 99, pp. 253-262, 2005.

[7] K. Sumiyoshi, S. Yamada, H. Suzuki, H. Shen, S. Chiba, and H. Toki, "Postbounce evolution of core-collapse supernovae: longterm effects of the equation of state," Astrophysical Journal Letters, vol. 629, no. 2, pp. 922-932, 2005.

[8] A. Burrows, E. Livne, L. Dessart, C. D. Ott, and J. Murphy, "A new mechanism for core-collapse supernova explosions," Astrophysical Journal Letters, vol. 640, no. 2, pp. 878-890, 2006.

[9] F. S. Kitaura, H. T. Janka, and W. Hillebrandt, "Explosions of $\mathrm{O}-\mathrm{Ne}-\mathrm{Mg}$ cores, the Crab supernova and subluminous type IIP supernovae," Astronomy and Astrophysics, vol. 450, no. 1, pp. 345-350, 2006

[10] A. Burrows, L. Dessart, E. Livne, C. D. Ott, and J. Murphy, "Simulations of magnetically driven supernova and hypernova explosions in the context of rapid rotation," The Astrophysical Journal, vol. 664, no. 1, p. 416, 2007.

[11] S. W. Bruenn, A. Mezzacappa, W. R. Hix et al., "The explosion of a rotating star as a supernova mechanism," in American Institute of Physics Conference Series, G. Giobbi, A. Tornambe, G. Raimondo et al., Eds., vol. 1111, pp. 593-601, 2009.

[12] A. Marek and H. T. Janka, "Delayed neutrino-driven supernova explosions aided by the standing accretion-shock instability," Astrophysical Journal Letters, vol. 694, no. 1, pp. 664-696, 2009.

[13] Y. Suwa, K. Kotake, T. Takiwaki, S. C. Whitehouse, M. Liebendörfer, and K. Sato, "Explosion geometry of a rotating $13 \mathrm{~m} \odot$ star driven by the sasi-aided neutrino-heating supernova mechanism," Publications of the Astronomical Society of Japan, vol. 62, no. 6, pp. L49-L53, 2010.

[14] T. Fischer, S. C. Whitehouse, A. Mezzacappa, F. K. Thielemann, and M. Liebendörfer, "Protoneutron star evolution and the neutrino-driven wind in general relativistic neutrino radiation hydrodynamics simulations," Astronomy \& Astrophysics, vol. 517, article A80, 25 pages, 2010.
[15] T. Takiwaki, K. Kotake, and Y. Suwa, “Three-dimensional hydrodynamic core-collapse supernova simulations for an $11.2 M_{\odot}$ star with spectral neutrino transport," Astronomical Journal, vol. 738 , no. 2, article 165,13 pages.

[16] G. S. Bisnovaty-Kogan, "Mechanism of core-collapse supernovae \& simulation results from the CHIMERA code," Astronomicheskii Zhurnal, vol. 47, p. 813, 1970.

[17] N. V. Ardeljan, G. S. Bisnovatyi-Kogan, and S. G. Moiseenko, "Explosion mechanisms of supernovae: the magnetorotational model," Physics-Uspekhi, vol. 40, pp. 1076-1079, 1997.

[18] N. V. Ardeljan, G. S. Bisnovatyi-Kogan, and S. G. Moiseenko, "Magnetorotational mechanism: 2D simulation," in The Local Bubble and Beyond Lyman-Spitzer-Colloquium: Proceedings of the IAU Colloquium No. 166, vol. 506 of Lecture Notes in Physics, pp. 145-148, 1998.

[19] G. S. Bisnovatyi-Kogan, N. V. Ardeljan, and S. G. Moiseenko, "Magnetorotational explosions: supernovae and jet formation," Memorie della Societa Astronomica Italiana, vol. 73, pp. 11341143, 2002.

[20] N. V. Ardeljan, G. S. Bisnovatyi-Kogan, and S. G. Moiseenko, "Magnetorotational supernovae," Monthly Notices of the Royal Astronomical Society, vol. 359, no. 1, pp. 333-344, 2005.

[21] S. G. Moiseenko, G. S. Bisnovatyi-Kogan, and N. V. Ardeljan, "A magnetorotational core-collapse model with jets," Monthly Notices of the Royal Astronomical Society, vol. 370, no. 1, pp. 501$512,2006$.

[22] G. S. Bisnovatyi-Kogan, S. G. Moiseenko, and N. V. Ardeljan, "Different magneto-rotational supernovae," Astronomy Reports, vol. 12, no. 12, pp. 997-1008, 2008.

[23] K. Kotake, H. Sawai, S. Yamada, and K. Sato, "Magnetorotational effects on anisotropic neutrino emission and convection in core-collapse supernovae," Astrophysical Journal Letters, vol. 608, no. 1, pp. 391-404, 2004.

[24] K. Kotake, S. Yamada, K. Sato, K. Sumiyoshi, H. Ono, and H. Suzuki, "Gravitational radiation from rotational core collapse: effects of magnetic fields and realistic equations of state," Physical Review D, vol. 69, no. 12, Article ID 124004, 2004.

[25] S. Yamada and H. Sawai, "Numerical study on the rotational collapse of strongly magnetized cores of massive stars," Astrophysical Journal Letters, vol. 608, no. 2, pp. 907-924, 2004.

[26] S. Yamada, K. Kotake, and T. Yamasaki, “The role of neutrinos, rotations and magnetic .elds in collapse-driven supernovae," New Journal of Physics, vol. 6, article 79, pp. 1-24, 2004.

[27] T. Takiwaki, K. Kotake, S. Nagataki, and K. Sato, "Magnetodriven shock waves in core-collapse supernovae," Astrophysical Journal Letters, vol. 616, no. 2, pp. 1086-1094, 2004.

[28] J. M. Stone and M. L. Norman, "ZEUS-2D: a radiation magnetohydrodynamics code for astrophysical flows in two space dimensions. II: the magnetohydrodynamic algorithms and tests," Astrophysical Journal Supplement, vol. 80, p. 791, 1992.

[29] H. Shen, H. Toki, K. Oyamatsu, and K. Sumiyoshi, "Relativistic equation of state of nuclear matter for supernova and neutron star," Nuclear Physics A, vol. 637, no. 3, pp. 435-450, 1998.

[30] S. Nishimura, K. Kotake, M. A. Hashimoto et al., "r-process nucleosynthesis in magnetohydrodynamic jet explosions of core-collapse supernovae," Astrophysical Journal Letters, vol. 642, no. 1, pp. 410-419, 2006.

[31] M. Liebendörfer, A. Mezzacappa, F. K. Thielemann, B. Messer, W. R. Hix, and S. Bruenn, "Probing the gravitational well: no supernova explosion in spherical symmetry with general relativistic Boltzmann neutrino transport," Physical Review D, vol. 63, no. 10, Article ID 103004, 13 pages, 2001. 
[32] C. Winteler, R. Käppeli, A. Perego et al., "Magnetorotationally driven supernovae as the origin of early galaxy r-process elements?" Astrophysical Journal Letters, vol. 750, no. 1, article L22, 2012.

[33] M. Saruwatari, M. Hashimoto, K. Kotake, and S. Yamada, "R-process nucleosynthesis during the magnetohydrodynamics explosions of a massive star," in Proceedings of the 10th Internal Symposium on Origin of Matter and Evolution of Galaxies (OMEG '10), vol. 1269 of AIP Conference Proceedings, pp. 409411, Osaka, Japan, March, 2010.

[34] K. Mori, E. Gotthelf, S. Zhang et al., "NuSTAR discovery of A $3.76 \mathrm{~s}$ transient magnetar near sagittarius A," The Astrophysical Journal, vol. 770, no. 2, article L23.

[35] R. Epstein, "The generation of gravitational radiation by escaping supernova neutrinos," The Astrophysical Journal, vol. 223, pp. 1037-1042, 1978.

[36] M. Ruffert, H. T. Janka, and G. Schafer, "Coalescing neutron stars-a steptowards physical models I. Hydrodynamical evolution and gravitational-wave emission," Astronomy \& Astrophysics, vol. 311, pp. 532-566, 1996.

[37] A. Staudt and H. V. Klapdor-Kleingrothaus, "Calculation of beta-delayed fission rates of neutron rich nuslei far off stability," Nuclear Physics A, vol. 549, no. 2, pp. 254-264, 1992.

[38] K. A. van Riper and J. M. Lattimer, "Stellar core collapse. I: infall epoch," The Astrophysical Journal, vol. 249, pp. 270-289, 1981.

[39] K. Kotake, S. Yamada, and K. Sato, "Anisotropic neutrino radiation in rotational core collapse," Astrophysical Journal Letters, vol. 595, no. 1, pp. 304-316, 2003.

[40] S. Rosswog and M. Liebendörfer, "High-resolution calculations of merging neutron stars. II: neutrino emission," Monthly Notices of the Royal Astronomical Society, vol. 342, no. 3, pp. 673689.

[41] K. A. van Riper, "Stellar core collapse. II: inner core bounce and shock propagation," The Astrophysical Journal, vol. 257, no. 15, pp. 793-820, 1982.

[42] H. A. Bethe, "Supernova mechanisms," Reviews of Modern Physics, vol. 62, no. 4, pp. 801-866, 1990.

[43] H. T. Janka, "Conditions for shock revival by neutrino heating in core-collapse supernovae," Astronomy and Astrophysics, vol. 368 , no. 2, pp. 527-560, 2001.

[44] D. L. Tubbs and D. N. Schramm, "Neutrino opacities at high temperatures and densities," The Astrophysical Journal, vol. 201, pp. 467-488, 1975.

[45] R. I. Epstein and C. J. Pethick, "Lepton loss and entropy generation in stellar collapse," The Astrophysical Journal, vol. 243, pp. 1003-1012, 1981.

[46] G. M. Fuller, W. A. Fowler, and M. J. Newmann, "Stellar weak interaction rates for intermediate-mass nuclei. II: $\mathrm{A}=21$ to $\mathrm{A}=$ 60," The Astrophysical Journal, vol. 252, pp. 715-740, 1982.

[47] K. Langanke, G. Martínez-Pinedo, J. M. Sampaio et al., "Electron capture rates on nuclei and implications for stellar core collapse," Physical Review Letters, vol. 90, no. 24, Article ID 241102, pp. 1-4, 2003.

[48] S. L. Shapiro and S. A. Teukolsky, Black Holes, White Dwarfs, and Neutron Stars, Wiley, 1983.

[49] S. A. Bludman, I. Lichtenstadt, and G. Hayden, "Homologous collapse and deleptonization of an evolved stellar core," The Astrophysical Journal, vol. 261, pp. 661-676, 1982.

[50] J. Cooperstein, "Neutrinos in supernovae," Physics Reports, vol. 163, no. 1-3, pp. 95-126, 1988.
[51] A. Heger, S. E. Woosley, and H. C. Spruit, "Presupernova evolution of differentially rotating massive stars including magnetic fields," Astrophysical Journal, vol. 626, no. 1, pp. 350-363, 2005.

[52] D. D. Clayton, Principles of Stellar Evolution and Nucleosynthesis, McGraw-Hill, New York, NY, USA, 1968.

[53] W. A. Fowler and F. Hoyle, "Neutrino processes and pair formation in massive stars and supernovae," Astrophysical Journal Supplement, vol. 9, p. 201, 1964.

[54] L. F. Roberts, S. Reddy, and G. Shen, "Medium modification of the charged-current neutrino opacity and its implications," Physical Review C, vol. 86, no. 6, Article ID 065803, 10 pages, 2012.

[55] G. Martinez-Pinedo, T. Fischer, A. Lohs, and L. Huther, "Charged-current weak interaction processes in hot and dense matter and its impact on the spectra of neutrinos emitted from proto-neutron star cooling," Physical Review Letters, vol. 109, no. 25, Article ID 251104, 2012.

[56] M. Liebendörfer, S. C. Whitehouse, and T. Fischer, "The isotropic diffusion source approximation for supernova neutrino transport," Astrophysical Journal Letters, vol. 698, no. 2, pp. 11741190, 2009.

[57] M. Ono, M. A. Hashimoto, S. I. Fujimoto, K. Kotake, and S. Yamada, "Explosive nucleosynthesis in magnetohydrodynamical jets from collapsars," Progress of Theoretical Physics, vol. 122, no. 3, pp. 755-777, 2009.

[58] H. T. Janka and E. Müller, "Neutrino heating, convection, and the mechanism of Type-II supernova explosions," Astronomy \& Astrophysics, vol. 306, p. 167, 1996.

[59] Y. Suwa, K. Kotake, T. Takiwaki, M. Liebendorfer, and K. Sato, "Impacts of collective neutrino oscillations on supernova explosions," Astronomical Journal, vol. 749, no. 2, article 98, 17 pages.

[60] S. I. Fujimoto, K. Kotake, M. A. Hashimoto, M. Ono, and N. Ohnishi, "Explosive nucleosynthesis in the neutrino-driven aspherical supernova explosion of a non-rotating $15 \mathrm{M} \infty$ star with solar metallicity," Astrophysical Journal, vol. 738, no. 1, article 61, 2011

[61] S. I. Fujimoto, K. Kotake, S. Yamada, M. A. Hashimoto, and K. Sato, "Magnetohydrodynamic simulations of a rotating massive star collapsing to a black hole," Astrophysical Journal Letters, vol. 644, no. 2, pp. 1040-1055, 2006.

[62] M. Ono, S. Nagataki, H. Ito et al., "Matter mixing in aspherical core-collapse supernovae: a search for possible conditions for conveying $56 \mathrm{Ni}$ into high velocity regions," The Astrophysical Journal, vol. 773, no. 2, article 161, 2013.

[63] V. V. Usov, "Millisecond pulsars with extremely strong magnetic fields as a cosmological source of $\gamma$-ray bursts," Nature, vol. 357, pp. 472-474, 1992.

[64] C. Thompson, "A model of gamma-ray bursts," Monthly Notices of the Royal Astronomical Society, vol. 270, no. 3, p. 480, 1994.

[65] E. G. Blackman and I. Yi, "On fueling gamma-ray bursts and their afterglows with pulsars," Astrophysical Journal Letters, vol. 498, no. 1, pp. L31-L35, 1998.

[66] J. C. Wheeler, I. Yi, P. Höflich, and L. Wang, "Asymmetric supernovae, pulsars, magnetars, and gamma-ray bursts," The Astrophysical Journal, vol. 537, no. 2, pp. 810-823, 2000.

[67] B. Zhang and P. Meszaros, "Gamma-ray burst afterglow with continuous energy injection: signature of a highly magnetized millisecond pulsar," The Astrophysical Journal Letters, vol. 552, no. 1, article L35, 2001. 
[68] T. A. Thompson, P. Chang, and E. Quataert, "Magnetar spindown, hyperenergetic supernovae, and gamma-ray bursts," Astrophysical Journal Letters, vol. 611, no. 1, pp. 380-393, 2004.

[69] N. Bucciantini, E. Quataert, J. Arons, B. D. Metzger, and T. A. Thompson, "Magnetar-driven bubbles and the origin of collimated outflows in gamma-ray bursts," Monthly Notices of the Royal Astronomical Society, vol. 380, no. 4, pp. 1541-1553, 2007.

[70] N. Bucciantini, E. Quataert, J. Arons, B. D. Metzger, and T. A. Thompson, "Relativistic jets and long-duration gamma-ray bursts from the birth of magnetars," Monthly Notices of the Royal Astronomical Society, vol. 383, no. 1, pp. L25-L29, 2008.

[71] N. Bucciantini, E. Quataert, B. D. Metzger, T. A. Thompson, J. Arons, and L. Del Zanna, "Magnetized relativistic jets and longduration GRBs from magnetar spin-down during core-collapse supernovae," Monthly Notices of the Royal Astronomical Society, vol. 396, no. 4, pp. 2038-2050, 2009.

[72] B. D. Metzger, T. A. Thompson, and E. Quataert, "Proto-neutron star winds with magnetic fields and rotation," Astrophysical Journal Letters, vol. 659, no. 1, pp. 561-579, 2007. 

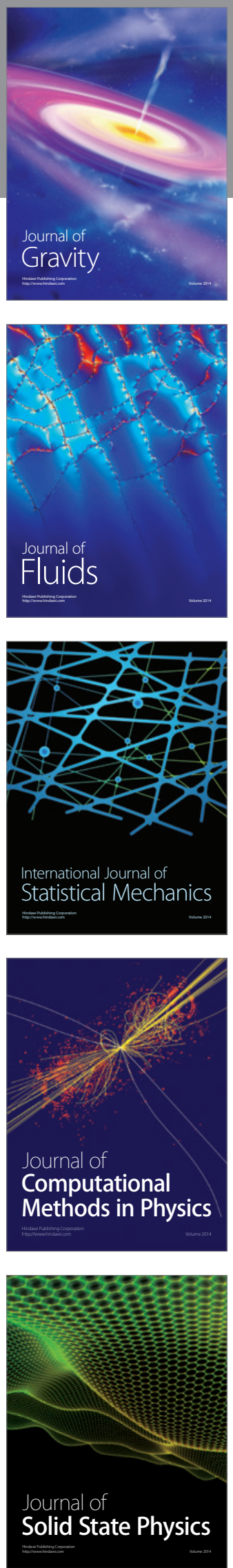

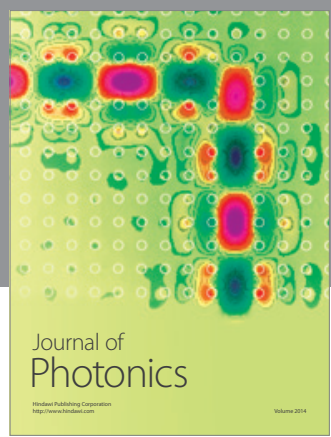

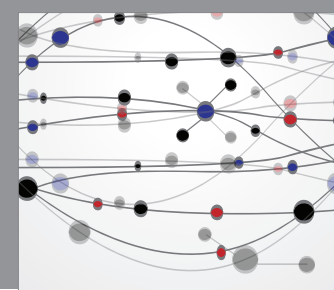

The Scientific World Journal

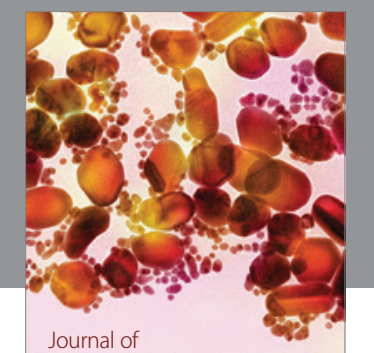

Soft Matter
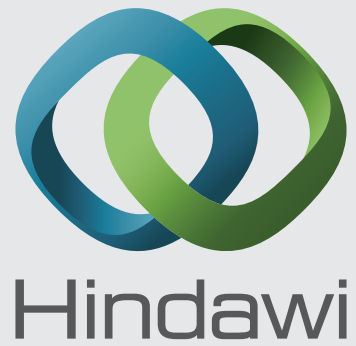

Submit your manuscripts at

http://www.hindawi.com
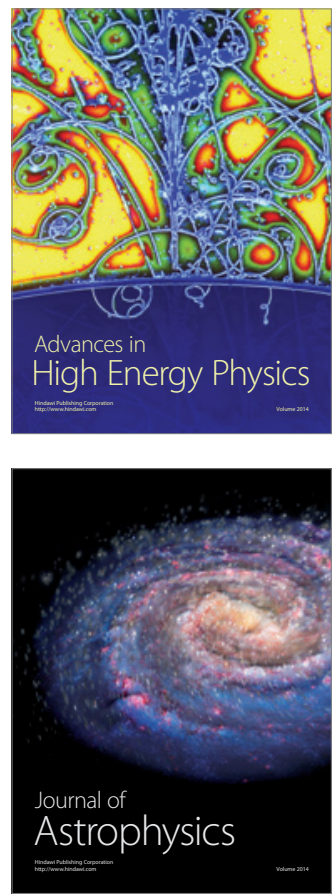
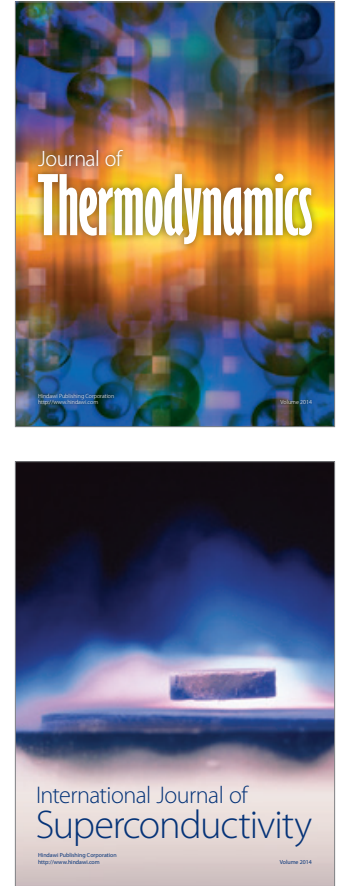
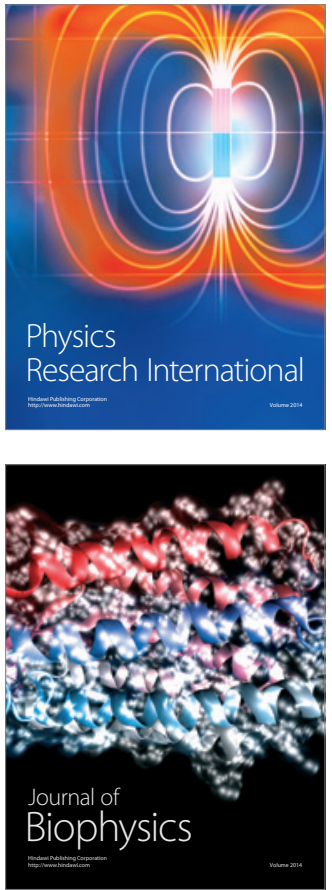
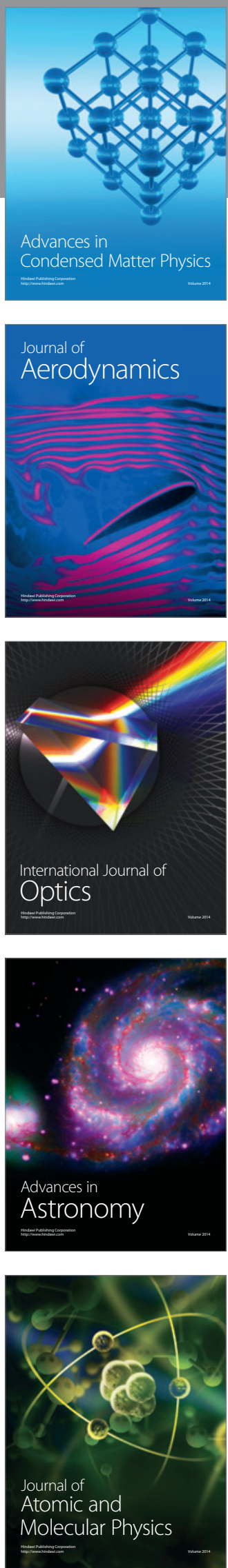